\title{
Equipo Didáctico para Aprendizaje Colaborativo en Automatización e Informática Industrial
}

\author{
Juan V. Martín ${ }^{1}$, Fernando Tadeo ${ }^{2}$, Teresa Álvarez ${ }^{2}$ y Jesús Peláez ${ }^{1}$ \\ (1) Universidad de Burgos, Escuela Politécnica Superior, Dpto. Ingeniería Electromecánica, \\ Avda. Cantabria s/n, 09006 Burgos-España (e-mail: jvmartin@ubu.es, jpelaez@ubu.es) \\ (2) Universidad de Valladolid, Escuela de Ingenierías Industriales, Dpto. Ingeniería de Sistemas y \\ Automática, Valladolid-España (e-mail: fernando@autom.uva.es, tere@autom.uva.es)
}

\section{Resumen}

En este trabajo se presenta un equipo didáctico desarrollado en la Universidad de Burgos en España, utilizado para impartir docencia de laboratorio en las asignaturas de automatización e informática industrial, con la metodología de aprendizaje colaborativo. El equipo está diseñado usando una arquitectura abierta y flexible, tomando en cuenta el ambiente industrial real y la posibilidad de trabajar en grupos, cumpliendo cada alumno diferentes roles. Se muestra que el uso de este equipo en aprendizaje activo aumenta la motivación de los estudiantes y mejora los resultados, con más estudiantes que logran los objetivos del curso. Además, a través de una encuesta se conoce la satisfacción del alumno con la metodología utilizada.

\section{Didactic Equipment for Collaborative Learning in Industrial Automation and Informatics}

\begin{abstract}
This paper presents a didactic piece of equipment developed at the University of Burgos in Spain for the teaching-learning process of modern industrial automation and industrial informatics in a collaborative learning environment. The equipment is designed using an open and flexible architecture, taking into account the real industrial environment and the possibility of working in groups, in which students play different roles. It is shown that the use of this kind of equipment in active learning environments increases the motivation of the students and improves the results, with more students achieving the learning objectives. Moreover, the evaluation of the students through anonymous surveys shows the good acceptance of the students of this novel learning technique.
\end{abstract}

Keywords: collaborative learning, automation, industrial informatics, Scada systems 


\section{INTRODUCCIÓN}

La finalidad del proceso educativo es proporcionar a nuestras generaciones de jóvenes los conocimientos necesarios que les permitan desenvolverse satisfactoriamente en la sociedad que les ha tocado vivir. La formación que reciben, a medida que van cursando los distintos niveles del proceso educativo, ha de prepararles para la vida y debe integrar la recreación del significado de las cosas, la cooperación, la discusión, la negociación y la resolución de todo tipo de problemas.

El actual proceso de enseñanza-aprendizaje está basado en un modelo centrado en la enseñanza, donde el profesor es el actor principal y el alumno, en un elevado número de casos, es un receptor pasivo. Este modelo, con una metodología expositiva, fomenta la adquisición del conocimiento y muy especialmente la memoria y la comprensión, y el acceso al conocimiento normalmente es a través del profesor.

La firma de la Declaración de Bolonia en1999, es consecuencia de la importancia de un desarrollo armónico de un Espacio Europeo de Educación Superior (EEES) antes del año 2010. Este objetivo está propiciando diversos foros de debate en todos los países miembros, para la evaluación de programas académicos convergentes que aseguren una calidad docente, adoptando el sistema de transferencia de créditos ECTS (European Credit Transfer System). Esto permitirá un reconocimiento académico inmediato de títulos, una movilidad entre países y un aprendizaje a lo largo de toda la vida. Desde un punto de vista exclusivamente docente, la firma de dicha Declaración supone un cambio en la manera de hacer las cosas para la gran mayoría del profesorado actual de nuestras Universidades Españolas. Este cambio se debe al nuevo modelo de Enseñanza-Aprendizaje en el ámbito del EEES, que defiende un modelo centrado en el estudiante, en el que se favorece su actividad y protagonismo; y persigue desarrollar diferentes capacidades trasversales y preparar al estudiante para el life-long learning. Estos nuevos planteamientos suponen un cambio en la planificación de la impartición docente de las distintas materias, y sobre todo, en lo relativo a objetivos de aprendizaje, trabajo del estudiante, metodologías activas y evaluación (Prince, 2004; Prince y Felder, 2006; Rodríguez, 2009).

Dentro del abanico de metodologías activas, se encuentra el aprendizaje colaborativo, caracterizado por rechazar la observación pasiva, la repetición y la memorización para promover la confrontación de opiniones, el compartir conocimientos, el liderazgo múltiple, y la multidisciplinariedad (Carrió, 2007). Según Gros (1997), la función del profesor es apoyar las decisiones del alumno, siendo los alumnos quienes desarrollan sus propias estrategias de aprendizaje, señalan sus objetivos y metas, al mismo tiempo que se responsabilizan de qué y cómo aprender. Cada miembro del equipo, entendiendo por equipo a un conjunto de personas que trabajan para alcanzar un fin común mediante acciones colaborativas, asume su papel dentro del grupo, como líder de los conocimientos que se le han asignado, aunque cada uno comprende que el grupo necesita de él para completar los conceptos que el grupo desea conocer. Cada participante, aporta lo mejor de sí para que el grupo consiga un beneficio, consiguiéndose que se establezca una relación de interdependencia que favorece la autoestima de los participantes y las relaciones interpersonales dentro del grupo. Para no correr el riesgo de promover experiencias caracterizadas por actitudes individualistas, que pueden dificultar entablar una interacción favorable que conduzca a la interdependencia positiva, se aconseja atender el proceso desde la conformación misma del equipo (Calzadilla, 2002).

El aprendizaje colaborativo es una propuesta de enseñanza-aprendizaje basada en los conceptos de cooperación, trabajo en equipo, comunicación y responsabilidad. Cada integrante participa para obtener unas conclusiones que se desprenden de la aportación de cada individuo para llegar a un acuerdo en un tema. Todos los miembros del grupo son líderes y evaluadores de los conceptos que se exponen, y aunque exista un coordinador del grupo, no actúa en ningún momento como líder, sino que cada uno actúa como líder de la tarea que le ha asignado su grupo. Cada responsable de una tarea después la expone al equipo que actúa como evaluador, por ello la comunicación es de vital importancia en este tipo de aprendizaje (Carrió ,2007). 
Según indican autores tales como Díaz-Barriga y Hernández (2002), el aprendizaje colaborativo se caracteriza por la igualdad que debe tener cada individuo en el proceso de aprendizaje y la mutualidad, entendida como la conexión, profundidad y bidireccionalidad que alcance la experiencia, siendo ésta una variable en función del nivel de competitividad existente, la distribución de responsabilidades, la planificación conjunta y el intercambio de roles para lograr una meta común.

Otra de las metodologías activas, es el aprendizaje cooperativo. Como explica Domingo (2008), trabajar con equipos reducidos de estudiantes aporta unas ventajas incuestionables a la docencia al ser una técnica de aprendizaje activo que permite al estudiante no tan sólo la oportunidad de alcanzar un aprendizaje significativo sino que le añade valores como la capacidad de poderse expresar tanto de forma oral como escrita, estructurar sus ideas, defenderlas, matizarlas, etc.

Para poner en práctica metodologías activas, con la intención de que el alumno aprenda y participe de forma activa en la construcción de su propio conocimiento, y esto le permita lograr los objetivos de aprendizaje, con este trabajo se muestra uno de los equipos didácticos desarrollados en la Universidad de Burgos, utilizado a la hora de impartir docencia de laboratorio en las asignaturas de Automatización e Informática Industrial, con la metodología de aprendizaje colaborativo, y que en este contexto se aplica como la metodología basada en que los estudiantes aprenden trabajando en equipo en la realización de una tarea común, siendo ellos mismos los responsables de su planificación y distribución de tareas. Aunque el equipo puede utilizarse en distintas materias, y con distintas metodologías activas, se presenta para la materia de Automatización Industrial, y con una aplicación concreta: la supervisión y el control de un variador industrial

Los motivos iniciales que impulsaron el desarrollo de estos equipos didácticos no fueron el poner en práctica metodologías de aprendizaje activo, si no mejorar la calidad docente. Sin embargo, aprovechando que el nuevo modelo de enseñanza-aprendizaje tenía prevista su implantación para el curso académico 2010/2011, se consideró conveniente iniciar un proceso de cambio progresivo, para la adaptación de las asignaturas de Automatización e Informática Industrial al EEES. Con ello, se pretendía lograr una transición suave del modelo actual, centrado fundamentalmente en la enseñanza por parte del profesor, al modelo futuro, basado en el aprendizaje del alumno. A parte de evitar una ruptura brusca entre ambos modelos, otra de las razones que justificaban el inicio del cambio, eran la falta de asistencia a clase, baja tasa de cumplimiento de objetivos de aprendizaje y la pasividad mostrada por los alumnos, en los cursos anteriores al 2005/2006.

Al indagar las razones que habían llevado a esta situación (en base a encuestas anónimas) se identificaron como posibles causas la poca participación activa del alumno, y la no percepción por parte del alumno de la conexión existente entre los conocimientos adquiridos y el entorno industrial al que van los alumnos egresados. En efecto, en el planteamiento original de la organización docente la participación de los alumnos era escasa y los equipos utilizados no facilitaban el desarrollo de prácticas cercanas a la realidad industrial. Por ello, además de perseguir una transición suave entre los modelos expuestos, se planteó como objetivo de la experiencia que se presenta en este trabajo el aumentar la participación activa del alumno, disminuir el porcentaje de alumnos no presentados al examen, mejorar el rendimiento académico, impulsar el trabajo en equipo, favorecer las relaciones interpersonales, y poner en práctica la metodología de aprendizaje colaborativo.

Para lograr los objetivos expuestos, se están utilizando en la actualidad en la Universidad de Burgos los equipos didácticos desarrollados, cuyo diseño permite que el alumno trabaje activamente, se relacione y potencie sus habilidades en un entorno cercano al de su futuro profesional, al ofrecerle la posibilidad de aprender en un ámbito más cercano a la realidad industrial actual.

De la revisión de trabajos similares al presentado en el artículo, cabe señalar el trabajo de Benítez et al. (2004) que utiliza la metodología de aprendizaje cooperativo basado en problemas en el diseño y desarrollo de proyectos de ingeniería, y presenta un caso práctico de una experiencia 
realizada con la asignatura de automatización industrial. También destacamos el trabajo de Domínguez et al. (2005) que trabajando con estrategias docentes colaborativas basadas en la utilización de laboratorios remotos vía Internet, abren la puerta al trabajo colaborativo basado en el uso de equipos industriales deslocalizados. Del mismo modo se resalta el trabajo de Gómez et al. (2006) cuya característica fundamental es el empleo de contenidos integrados teórico-prácticos en soporte electrónico y el desarrollo de prácticas reales en un entorno docente presencial, con supervisión y control por parte del profesor.

Estas ideas también se comparten en el documento "Propuestas para la renovación de las metodologías educativas en la universidad" (MEC, 2006), dentro de la estrategia general de renovación de las metodologías. En la misma línea Álvarez et al. (2008), trabajan con la metodología Aprender Enseñando, facilitando el aprendizaje de sus alumnos mediante la elaboración de materiales didácticos para aprendizaje autónomo de otros alumnos.

\section{DESCRIPCIÓN DEL EQUIPO DIDÁCTICO}

De la revisión de los equipos didácticos comerciales actuales, se puede comprobar que existen excelentes equipos didácticos comerciales en el ámbito de la automatización industrial, como se muestra en la figura 1. Sin embargo, se observa que estos equipos presentan principalmente dos dificultades a tener en cuenta, desde una perspectiva exclusivamente docente (Martín y Tadeo, 2007): Se comercializan como una solución docente, sin permitir mostrar a los alumnos las interioridades de su diseño, y la mayoría carecen de un enfoque cercano a la realidad industrial.

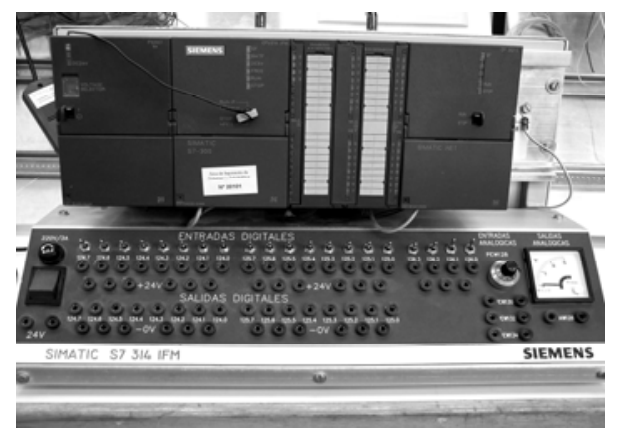

Fig. 1: Ejemplo de equipo didáctico comercial

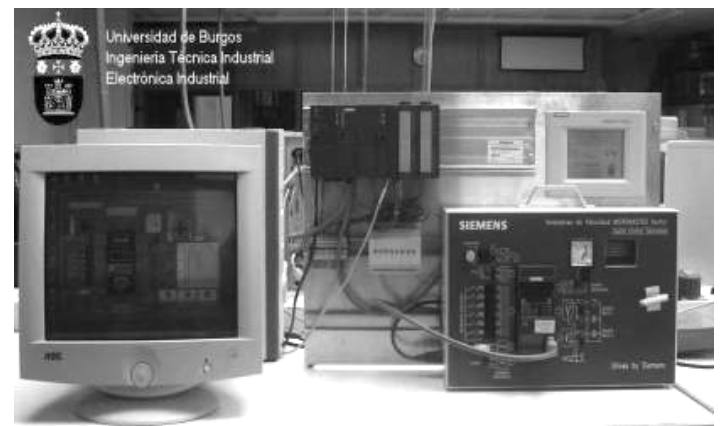

Fig. 2: Equipo didáctico desarrollado

La arquitectura que presenta el equipo didáctico desarrollado (ver figura 2), está basada en la configuración que se puede encontrar en los sistemas automatizados de la mayor parte de los procesos industriales actuales. Esta característica, permite utilizarlo para crear escenarios de aprendizaje en el ámbito de la Empresa, y así contribuir a mejorar la formación de nuestros alumnos al ofrecerles la posibilidad de aprender en un ámbito más cercano a la realidad industrial actual.

El equipo didáctico se compone de un panel principal o de mando, de un panel operativo y de un sistema SCADA (figura 3). Esta arquitectura también persigue como objetivo poder enseñar a aprender con la práctica, los conceptos de parte de mando o control, parte operativa, y sistemas SCADA; y todo ello desarrollado mediante aprendizaje activo.

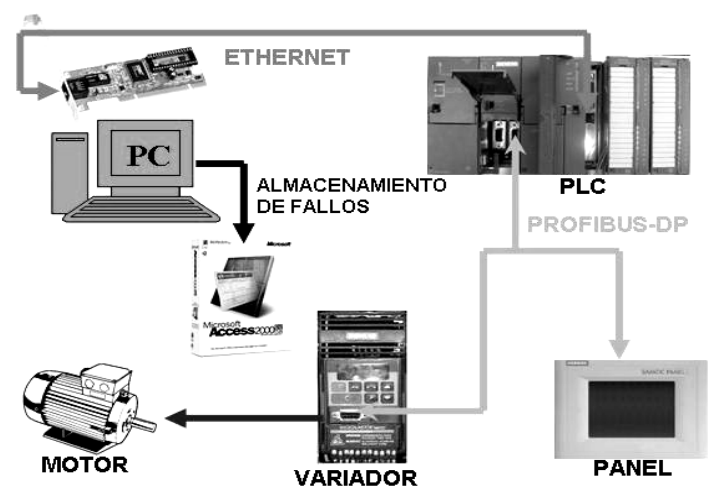

Fig. 3: Componentes del equipo didáctico 


\section{El panel de mando}

Es el equivalente al armario de la instalación de un sistema automatizado (Piedrafita, 2004), como el que se pueden encontrar en la mayoría de los procesos industriales actuales.

\section{El panel operativo}

A grandes rasgos se compone de un motor trifásico gobernado mediante un variador de frecuencia conectado con el autómata de la instalación a través del bus de campo Profibus DP (Felser, 2002; Piedrafita, 2004; Weigmann y Kilian, 2003). En las instalaciones reales, se encuentran aplicaciones similares en el control de cintas de transporte, pórticos manipuladores, y en la mayoría de las aplicaciones en las que se requiera el control de motores industriales a distintas velocidades.

\section{EI Sistema SCADA}

Como es bien conocido el acrónimo SCADA procede de las siglas "Supervisory Control And Data Acquisition" (Rodríguez, 2007). Se trata de una aplicación software especialmente diseñada para trabajar sobre ordenadores en el control de procesos, proporcionando información de los dispositivos de campo permitiendo supervisar y controlar el proceso desde el o los PCs en los que se encuentre instalada. En el caso del presente estudio, incluye el desarrollo, en el lenguaje de programación Visual Basic, de una Aplicación Didáctica que permite monitorizar, parametrizar y controlar un variador, junto con el motor trifásico que gobierna. Además permite la gestión y almacenamiento, en una base de datos Access, de los fallos ocurridos en la instalación. Contemplándose fallos externos simulados, a través de entradas digitales, y fallos internos provocados desde el propio variador.

Para poder acceder a las distintas funciones del sistema SCADA, la aplicación proporciona distintas pantallas, destacando las de Modo Supervisión, Modo Control y Gestión de Fallos.

\section{Modo supervisión}

Este modo permite la visualización de todos los parámetros del variador y actuar sobre él, como si se tratase del dispositivo real, pero sin posibilidad de cambiar ninguno de sus parámetros. Como puede apreciarse en la figura 4, también se visualiza, en la pantalla del PC, el estado de la parte operativa.

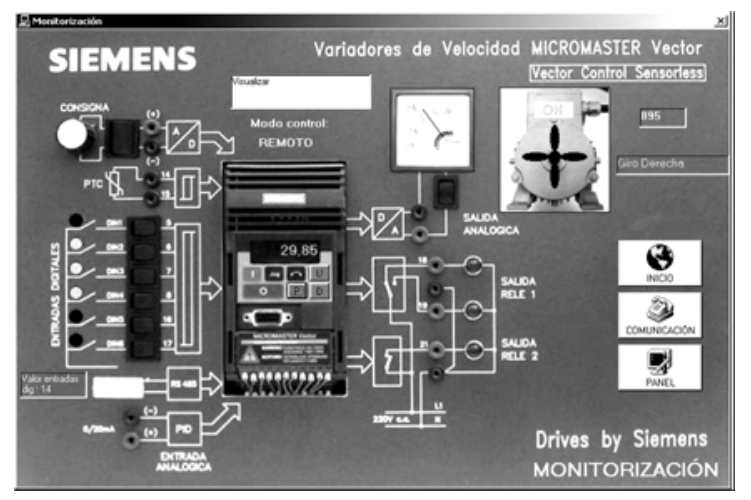

Fig. 4: Pantalla modo supervisión

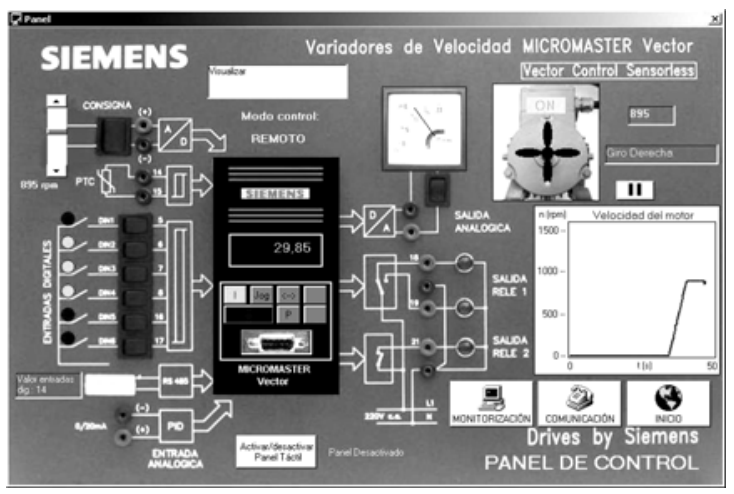

Fig. 5: Pantalla modo control

\section{Modo control}

Mediante el Modo Control (mostrado en la figura 5) no sólo se podrá gobernar la parte operativa, sino que también se podrá monitorizar y parametrizar. Con objeto de facilitar el aprendizaje del manejo del variador, la aplicación proporciona ciertas mejoras, respecto al dispositivo real: gráficas de velocidad, aceleración, deceleración, frenado, etc. 


\section{Gestión de fallos}

Para poder obtener históricos de averías e información de tiempos de parada de máquina la aplicación gestiona una base de datos almacenando información relativa al tipo de fallo, fecha, hora, etc. (ver figura 6).

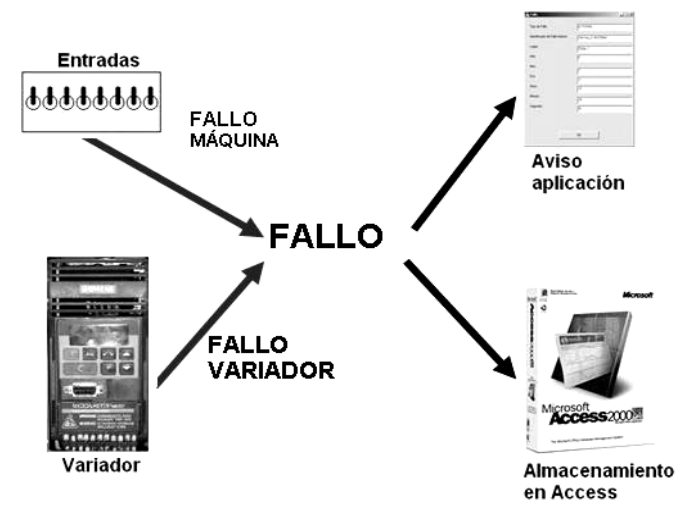

Fig. 6: Gestión de fallos

\section{METODOLOGÍA}

El desencadenante principal de la experiencia colaborativa surgió como consecuencia de la participación en el Proyecto de Investigación "Formación del Profesorado y Desarrollo de Experiencias Piloto en relación con la Convergencia Europea en las Enseñanzas de Ingeniería", financiado por la Consejería de Educación de la Junta de Castilla y León.

Esta experiencia de aprendizaje activo ha sido desarrollada en la Escuela Politécnica Superior de la Universidad de Burgos en la Titulación de Ingeniería Técnica Industrial, especialidad Electrónica Industrial; en la asignatura "Automatización Industrial", de tipo troncal, de segundo curso, segundo cuatrimestre, con una carga de 4.5 Créditos teóricos y 4.5 Créditos prácticos. La mayor parte de los alumnos de la asignatura, previamente han cursado la asignatura Informática Industrial del mismo curso, pero de primer cuatrimestre; si bien no todos la tienen superada. Esta característica provocará la formación de grupos heterogéneos. Por otro lado, la asignatura en sí es de integración de equipos y dispositivos, en la que el alumno necesita conocimientos previos de otras asignaturas. Sin embargo, el Plan de Estudios no contempla esta peculiaridad, y ello dificulta el aprendizaje de los estudiantes.

Como ya se ha comentado, con esta experiencia además de perseguir una transición suave entre el actual y el nuevo modelo de aprendizaje en el marco del EEES. Se planteó como objetivo el aumentar la participación activa del alumno, disminuir el porcentaje de alumnos no presentados al examen, mejorar el rendimiento académico, impulsar el trabajo en equipo, favorecer las relaciones interpersonales, y poner en práctica la metodología de aprendizaje colaborativo. De cara al profesorado, se marcó como objetivo el saber cuál es la valoración del profesor por parte de los alumnos, conocer mejor los mecanismos que dificultan el aprendizaje de los alumnos, para poder combatirlos de la forma adecuada, y a la vez aprovechar la experiencia para desarrollar habilidades relacionadas con el aprendizaje activo. Para poder extraer conclusiones de esta experiencia piloto de aprendizaje colaborativo, se necesitan exponer las metodologías utilizadas a lo largo de los diferentes cursos académicos, tanto en lo referente al desarrollo de la materia, como a criterios de evaluación.

\section{Metodología usada hasta el curso 2005/2006}

El método utilizado para desarrollar el aprendizaje de la asignatura, fue la exposición magistral de la materia y la realización de problemas tipo en el aula, por parte del profesor. La mayoría de los ejercicios propuestos para realizar fuera de clase, también eran resueltos por el profesor en el aula. Para la realización de las prácticas de laboratorio, se formaban parejas de alumnos, se les asignaban equipos didácticos comerciales de características similares al mostrado en la figura 1, y se les suministraba una guía con los pasos que debían llevar a cabo, en el desarrollo de cada una de las prácticas. En lo referente a la evaluación, se utilizó el procedimiento habitual de evaluación 
de la mayoría de las escuelas de ingeniería (Akar et al., 2004), y durante este periodo se hizo un examen teórico-práctico sobre los conocimientos técnicos de la materia.

\section{Metodología usada a partir del curso 2005/2006}

Debido a la escasa participación del alumno en su proceso de aprendizaje, y a las recomendaciones institucionales animando a un cambio de metodología centrada en el alumno, es necesario establecer una nueva metodología en la que el alumno participe activamente en su proceso de aprendizaje, y además de los conocimientos específicos de la materia adquiera otra serie de competencias que le preparen para el aprendizaje a lo largo de la vida (Rodríguez, 2009).

La metodología seleccionada combina la lección magistral, el aprendizaje cooperativo y el aprendizaje colaborativo. La lección magistral se utiliza para aquellos conceptos, que por su dificultad y complejidad exigirían al alumno demasiado tiempo y esfuerzo (Brown y Atkins, 1998; Humphreys et al., 2001). La planificación del aprendizaje está fuertemente guiado por el profesor, siendo esto una característica del aprendizaje cooperativo (Dillenbourg, 2000; Ballantine y McCourt, 2007). Sin embargo, en este caso no hay reparto de tareas, por parte del profesor entre los miembros del equipo, sino que es el grupo quien se organiza dividiendo el trabajo, realizando el reparto de tareas y responsabilidades, o trabajando juntos para posteriormente, mediante el diálogo y la negociación, llegar al consenso en la consecución final de los objetivos marcados, lo que es un rasgo característico del aprendizaje colaborativo (Carrio, 2007). En cuanto a la formación de grupos de trabajo, se establece como condición que el número de miembros del grupo sea de 4 personas, y se opta por dejar libertad a los alumnos para elegir a sus compañeros de trabajo, en un plazo máximo de 15 días.

Para la realización de las prácticas de laboratorio (PL), a cada uno de los grupos de trabajo formados, se les asigna un equipo didáctico de características similares al presentado en este trabajo. A continuación, con objeto de motivar a los alumnos y hacerles partícipes activos de su proceso de aprendizaje, se les muestra una aplicación didáctica lo más cercana posible a la realidad industrial actual, para que vean la utilidad y aplicabilidad de los conocimientos aprendidos de cara al mundo de la Empresa. Finalmente, se les pide que mediante trabajo colaborativo, con una estructura de organización empresarial, reproduzcan dicha aplicación, y a la vez se les invita a proponer alternativas, mejoras, etc., motivando así su creatividad, y calidad del trabajo desempeñado. Para seguir facilitando la participación activa del alumno dentro y fuera del aula, a todos los grupos se les asignan una serie de actividades de grupo (AG), para que sean resueltas mediante trabajo colaborativo. Además, para fomentar la cooperación y el trabajo en equipo, de cara a la presentación y evaluación de la actividad, el profesor designa al alumno responsable de hacer la presentación oral pública, de una determinada actividad. De igual modo, para favorecer el trabajo autónomo del alumno y valorar objetivamente sus conocimientos, se realiza un examen teórico-práctico (TP), sobre los conocimientos técnicos de la materia.

Con esta metodología, se pretende mantener activo al alumno en el desarrollo de los objetivos de aprendizaje, que el alumno perciba la utilidad de su aprendizaje, y en todo momento lo conecte y relacione con la Empresa. Además, se persigue que el alumno adquiera habilidades en comunicación oral y escrita, búsqueda de información, relaciones interpersonales, responsabilidad, colaboración, etc. En cuanto a la evaluación, debe estar diseñada para recoger, en cada momento, información del proceso enseñanza-aprendizaje y orientar la marcha del mismo, cuando los resultados obtenidos no son los esperados. Según Rodríguez y Beltrán (1994), la evaluación tiene que estar centrada en las competencias que se desarrollan en la asignatura, y los criterios de valoración deben ser claros y públicos.

En el caso bajo estudio, para evaluar el aprendizaje, se establecen los siguientes criterios: el trabajo en grupo (NG = PL + AG), representa el $40 \%$ de la nota final. El $60 \%$ restante, se obtiene mediante el examen final (TP), sobre los conocimientos técnicos de la materia.

\section{RESULTADOS Y DISCUSIÓN}

La figura 7 muestra que el porcentaje de alumnos que no realizan las actividades necesarias para su evaluación (no presentados) ha disminuido significativamente con el uso de la nueva metodología. La figura 8 muestra que con el uso de la nueva metodología el porcentaje de 
alumnos que adquieren los objetivos de aprendizaje (aprobados) ha aumentado también significativamente (de menos de un $40 \%$ a cerca de un $80 \%$ ).

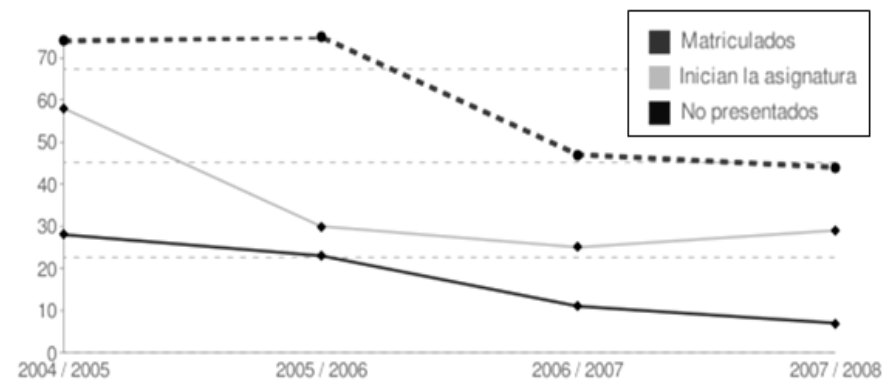

Fig. 7: Número de Matriculados y no presentados

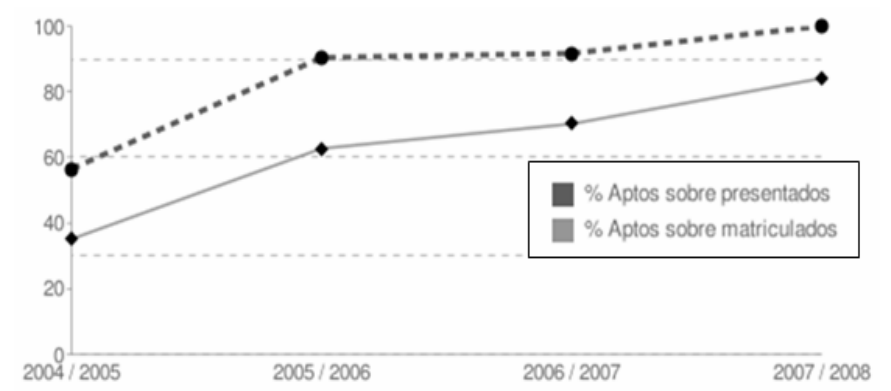

Fig. 8: Porcentaje de aptos

Para recabar la opinión de los alumnos sobre la metodología utilizada, con el fin de ajustar aquellos conceptos que el alumno considere deficientes, se realizó la encuesta individual que se muestra en la Tabla 1 , donde se detallan los valores medios y algunas de las contestaciones recibidas. De los valores medios obtenidos, se puede comprobar cómo la metodología de aprendizaje colaborativo (indicador 1 ) ha sido valorada muy positivamente, destacando la percepción por parte de los alumnos de la utilidad e importancia de las actividades realizadas de cara al futuro profesional (indicadores 2, 3 y 9). Del mismo modo, es importante señalar la satisfacción manifestada con el aprendizaje de la asignatura (indicador 6), y el trabajo aportado por los compañeros (indicador 10). Si bien en menor mediada, también los estudiantes consideran mejorada su capacidad de expresión oral y escrita (indicador 8), y las relaciones interpersonales (indicador 7). De las contestaciones recibidas, como dato a considerar, hay alumnos que demandan mayor peso del trabajo colaborativo sobre la nota final.

Por otra parte, en relación con la influencia específica sobre la mejora de resultados de cada aspecto que se ha cambiado en la forma de adquirir conocimientos (aprendizaje colaborativo, equipos didácticos, metodología de evaluación, etc.), puede comprobarse en la encuesta, como los alumnos consideran que la utilidad de las actividades realizadas, y su importancia de cara al futuro profesional han sido los aspectos más importantes (indicadores 3 y 9), aunque todos han sido valorados muy positivamente (como se comprueba en los indicadores 1-12, y en los comentarios cualitativos).

Tabla 1: Encuesta de satisfacción realizada (2008/2009).

(1= muy poco. $2=$ poco. $3=$ normal. $4=$ bastante $.5=$ mucho $)$

\begin{tabular}{|c|l|c|}
\hline & Indicador & Media \\
\hline 1 & ¿Cuál es tu valoración sobre la metodología de aprendizaje colaborativo? & 4.4 \\
\hline 2 & ¿Consideras de utilidad los contenidos de la asignatura? & 4.4 \\
\hline 3 & ¿Consideras de utilidad las actividades realizadas? & 4.7 \\
\hline 4 & ¿Esta metodología te ha ayudado a llevar al día la asignatura? & 4.0 \\
\hline 5 & ¿Recomendarías a un compañero esta asignatura? & 4.5 \\
\hline 6 & ¿Estás satisfecho con el aprendizaje de la asignatura? & 4.2 \\
\hline 7 & ¿Esta metodología ha propiciado nuevas relaciones con tus compañeros? & 3.9 \\
\hline 8 & ¿Consideras que ha mejorado tu expresión oral y escrita? & 3.8 \\
\hline 9 & ¿Qué importancia le das a la asignatura de cara al futuro profesional? & 4.6 \\
\hline 10 & ¿Estás satisfecho con el trabajo aportado por tus compañeros? & 4.3 \\
\hline 11 & ¿Consideras adecuada la evaluación de trabajos? & 3.6 \\
\hline 12 & ¿Cuál es la valoración del profesor? & 4.0 \\
\hline 13 & $\begin{array}{l}\text { Aspectos en contra de esta metodología: } \\
\text { - Que puede que alguien no trabaje lo suficiente, pero ya sabes tú con quién te has puesto. } \\
\text { - Requiere mucho tiempo, con respecto al resto de asignaturas. }\end{array}$ \\
\hline 14 & $\begin{array}{l}\text { Aspectos a favor de esta metodología: } \\
\text { - Se aprende mucho. Mucha colaboración, y puedes aprender de otros. } \\
\text { - Es una herramienta básica para el futuro profesional. Ayuda a trabajar en grupo. }\end{array}$ \\
\hline 15 & $\begin{array}{l}\text { Observaciones y sugerencias de mejora: } \\
\text { - Aumentar la importancia del trabajo en la nota en detrimento del examen. } \\
\text { - Resolución de más ejercicios prácticos en clase. Más prácticas. }\end{array}$ \\
\hline
\end{tabular}




\section{CONCLUSIONES}

De acuerdo con los objetivos propuestos, se presentan los avances logrados con la experiencia realizada. Debe destacarse que el porcentaje de alumnos presentados al examen se ha incrementado desde un valor del $62 \%$, con el método expositivo, a un valor del $85 \%$ utilizando la metodología presentada en este trabajo. La eficiencia de la metodología se ha reflejado en el porcentaje de aprobados, que ha pasado de un 35\% con el método expositivo, a un $84 \%$ con la nueva metodología.

En base a los resultados y a la propia experiencia, se puede afirmar que cuando el alumno percibe que los conocimientos que aprende y las actividades que realiza tienen aplicación directa de cara a su futuro profesional, aumenta su participación en las actividades de la asignatura y esto mejora sus resultados de aprendizaje.

La utilización de este equipo y otros similares, diseñados para que el alumno aprenda en escenarios que reproduzcan la realidad industrial actual, combinado con la metodología de aprendizaje colaborativo facilitan que el alumno además de aprender los conocimientos específicos de la materia adquiera otra serie de actitudes y valores preparándolo para el aprendizaje a lo largo de la vida.

\section{AGRADECIMIENTOS}

A todas aquellas personas que han colaborado en la realización de este equipo, y de forma especial a los miembros del proyecto UV25/05. De igual modo, se agradece la financiación a través del proyecto MiCInn DPI2007-66718-C04-02, y los comentarios de los revisores.

\section{REFERENCIAS}

Akar, E., E. Ozturk, B. Tuncer y M. Wiethoff; Evaluation of a collaborative virtual learning environment, Education and Training: 46(6/7), 343-352 (2004).

Álvarez, F., J.R. Rodríguez, E. Sanz y M. Fernández; Aprender Enseñando: Elaboración de Materiales Didácticos que facilitan el Aprendizaje Autónomo, Formación Universitaria: 1(6), 19-28 (2008).

Ballantine, J. y P. McCourt. Cooperative Learning: A Pedagogy to Improve Students' Generic Skills? Education \& Training: 49(2), 126-137 (2007).

Benítez, R., B. Giraldo y J. Domingo; Aprendizaje cooperativo basado en problemas en el diseño y desarrollo de proyectos de ingeniería. Actas del XII Congreso Universitario de Innovación Educativa en las Enseñanzas Técnicas. 12 CUIEET. Barcelona, España, 26 al 28 de Julio (2004).

Brown, G. y M. Atkins. Effective Teaching in Higher Education. Routledge (1998).

Calzadilla, M.; Aprendizaje colaborativo y tecnologías de la información y la comunicación. Revista Iberoamericana de Educación: 1, 10 páginas (2002). www.rieoei.org.

Carrió ML.; Ventajas del uso de la Tecnología en el Aprendizaje Colaborativo. Revista Iberoamericana de Educación: 41(4), 1-10 (2007). www.rieoei.org.

Díaz-Barriga, F. y G. Hernández; Estrategias docentes para un aprendizaje significativo. Una interpretación Constructivista. McGraw Hill (2002).

Dillenbourg, P.; What do you mean by Collaborative Learning, Collaborative Learning: Cognitive and Computational Approaches, Elsevier, Oxford (2000).

Domingo, J.; El aprendizaje cooperativo, Cuadernos de Trabajo Social: 21, 231-246 (2008). 
Domínguez, M., P. Reguera y J.J. Fuertes; Laboratorio Remoto para la Enseñanza de la Automática en la Universidad de León, Revista Iberoamericana de Automática e Informática Industrial: 2(2), 36-45 (2005).

Felser, M.; The Fieldbus Standards: History and Structures. Actas de Technology Leadership Day 2002, Organised by Microswiss Network, HTA Luzern, Suiza (2002).

Gómez, E. y otros cuatro autores; Metodología docente interactiva para el aprendizaje de tecnología de fabricación. I Jornadas de Innovación Educativa. Zamora, España, 20 al 22 de Junio (2006).

Gros, B.; Diseño y programas educativos. Pautas pedagógicas para la elaboración de software, Ariel, Barcelona (1997).

Humphreys, P., V. Lo, F. Chan y G. Duggan. Developing Transferable Groupwork Skills for Engineering Students, International Journal Engineering Education: 17(1), 59-66 (2001).

Martín, J.V. y F. Tadeo; An Open and Flexible Equipment for Learning Fieldbus Technology, Innovations 2007: World Innovations in Engineering Education and Research (2007).

MEC; Propuestas para la renovación de las metodologías educativas en la universidad, Ministerio de Educación y Ciencia (MEC), Consejo de Coordinación Universitaria; Secretaría General Técnica, Edigrafos, S.A. (2006).

Piedrafita, M.; Ingeniería de la Automatización Industrial. Ra-Ma (2004).

Prince, M.; Does Active Learning Work? A Review of the Research, Journal of Engineering Education: 93(3), 223-231 (2004).

Prince, M. y R.M. Felder; Inductive teaching and learning methods: Definitions, comparisons, and research bases, Journal of Engineering Education: 95(2), 123-138 (2006).

Rodríguez, J.L. y R. Beltrán. Evaluación del Currículo. Didáctica General: Un Enfoque Curricular. Editorial Marfil (1994).

Rodríguez, P.; Sistemas SCADA - Guía práctica. Marcombo (2007).

Rodríguez, R.M.; El reto de la convergencia europea: Necesidades y cambios, Revista de Formación e Innovación Educativa Universitaria: 2(1), 154-164 (2009).

Weigmann, J. y G. Kilian; Decentralization with Profibus DP/DPV1. Structure, configuration and use of Profibus DP with Simatic S7. Siemens (2003). 